\title{
Human Cognitive Features to Define Correlation Between Depression and Internet Gaming Disorder
}

\author{
Hari Mohan Pandey \\ Department of Computer Science \\ Edge Hill University \\ St Helens Rd, Ormskirk L39 4QP Lancashire, UK \\ Pandeyh@edgehill.ac.uk
}

\author{
Abhishek Jain \\ Computer Science and Engineering \\ Amity School of Engineering and Technology, Amity Uniersity \\ Noida, India \\ j.avishek@gmail.com
}

\begin{abstract}
This paper explores relationship between depression and internet gaming disorder (IGD) symptoms using electroencephalography (EEG) signals recordings. With the advancement of technology and penetration of Internet in human's life, gaming community also has shifted to online platform like massively multiplayer online role-playing games (MMORPGs) instead of physical games, causing multiple health hazards including behavioral and physical anomalies. Diagnosis of IGD is an arduous task using subjective assessments and need neurological methods to be used in identification. The umbrella of symptoms for IGD overlap with other neurological and behavioral disorders like depression, attention deficit hyperactivity disorder (ADHD), obsessivecompulsive disorder (OCD), thus can share the methods for their identification. In this paper, we determine the power spectral density (SPD) of EEG recording for depression subjects. Rigorous experiments are conducted to analyze the proposed system. Experimental results reveal the coherence between the power for the beta band for depression and IGD subjects. The results suggest that due to the comorbidity of IGD and depression correlation are cohesively predictive.
\end{abstract}

Keywords-Depression, EEG, Gaming Addiction, Internet Gaming Disorder, Major Depressive Disorder

\section{INTRODUCTION}

Human brain is a collection of millions of interconnected neurons which controls human behavior through different electromagnetic signals. Doctors and radiologists use different methods like electroencephalography (EEG) and magnetoencephalography (MEG) to acquire these signals for various studies. These signals have been used to analyze brain and its cognitive behavior to detect disorders in brain functioning. Neurological disorders especially behavioral disorders are hard to ascertain with traditional psychological methods that are used by psychologists, but it is crucial to discover them in early stage of a disease in order to reduce the global burden of disease [4]. Addiction and depression have been described as changes in someone's personality and behavior. It can also be effectively analysed by using brain waves. Addiction shows long-lasting use or dependence on some activity or substance. Generally, it is characterized by a repeated loss of control over dependency and craving towards it.

Gaming, specially over the internet is one of the interests among child and adolescents. Excessive gaming lead to the functional as well as structural changes in the brain areas which are involved cognitive features such as reward and punishment feelings. Playing games online gives feelings of pleasure and reward virtually rather than natural happiness to gamers and assumed by gamer as a way of relaxation (physical and mental) by voluntarily skipping routine activities of individual daily life. Majority of players are attracted by massively multiplayer online role-playing games (MMORPGs) where players are connected virtually mostly through their social network instead of their physical connections [12].

Digitalization and online activities including video games has elicited significant interest in new generation adolescent and adults. Gaming industry especially internet gaming is a booming market globally. Asia-pacific region is primary driver of continued growth for this industry. In year 2018, mobile games have $51 \%$ share which is expected to grow to $59 \%$ of global games market in 2021 [1]. Only in India, gaming market has recorded rapid growth in last decade and is currently around 290M USD industry and forecasting to become 1B USD industry by 2021 [11]. The forecast says that games market is expected to grow from $\$ 137.9$ billion in 2018 to $\$ 180.1$ billion in 2021 globally [1]. In one of the study was conducted in 2013, it has been found that IGD is more prevalent in Asian countries as compared to North America and Europe (APA, 2013) [5].

The term internet gaming disorder (IGD) was coined by world health organization (WHO) in their 11th release of international classification of diseases (ICD), which is also listed as diagnosable condition and research area scholars should focus on. WHO encouraged doctors and scholars to gain the evidence and unveil the solutions for such addictions. There are many overlapping symptoms among different neurological disorders. Few of them as subset are observed as: withdrawal, mood modification, salience, tolerance, conflict and relapse. These symptoms have been ccharacterized for the IGD [13].

Studies using traditional methods such as self-reports and questionnaire have not yielded results up to the mark. Thus, now a days more research is being done on neuroimaging and physiology of IGD. There is still debate among scholars on the inclusion criteria and diagnosis classification systems to properly categorize IGD as behavioral addiction.

For diagnosis of different behavioral disorder, most commonly used methods and frameworks referred by clinicians and practitioner are scale based psychological tests. These include different psychometric questionnaires, which are answered by patients and later analyzed by psychologist. These methods are human-intensive and result of these methods is highly influenced by psychologist experience. Another challenge which has been observed is due to fear of stigma patients does not speak and chances of getting correct answer become less. Hence, it leads to an inaccurate diagnosis [6]. With the emerging biomedical equipment more advanced sensors are available to use in the 
research work. Nowadays, numerous modalities are available for brain images or signals such as: (a) functional magnetic resonance imaging (fMRI); (b) computed tomography (CT); (c) positron emission tomography (PET); (d) electroencephalography (EEG); and (e) magnetoencephalography (MEG). Each of these methods have its own merits. Hence, sometimes combination of these hybrid modalities are used to discover complex disorders. Among these electroencephalography (EEG) and magnetoencephalography (MEG) are non-invasive and less painful methods which have almost no side effects and provide accurate interpretations helpful in diseases like autism, epilepsy, Alzheimer, etc. EEG offers higher temporal resolution than fMRI has a low cost. Therefore, it is suitable for small clinical and research environments.

EEG is a technique that measures electrical potential of signals from the brain using a special device, electroencephalogram. When brain cells (neurons) are activated the synaptic currents are produced within the dendrites, which generates a magnetic field measurable by electromyogram (EMG) machines and a secondary electrical field over the scalp measurable by EEG systems. Electrophysiological signals contain information about neural activities inside the brain at various frequencies, which can be helpful during the diagnosis or quantification of physiological states of brain.

TABLE I

FREQUENCY BANDS EXHIBTED BY BRAIN FUNCTIONS

\begin{tabular}{ll}
\hline \hline EEG Band & Brain Functions \\
\hline \hline Delta $(0-4 \mathrm{~Hz})$ & Appears in an adult's slow-wave sleep \\
Theta $(4-8 \mathrm{~Hz})$ & Found in Drowsiness and Sleepy state \\
Alpha $(8-12 \mathrm{~Hz})$ & Brain is in relaxed state \\
Beta $(12-30 \mathrm{~Hz})$ & Active thinking and cognitive functioning \\
Gamma $(30-70 \mathrm{~Hz})$ & These waves are observed during meditation \\
\hline \hline
\end{tabular}

With advancements in technology, advanced multichannel EEG devices are available with huge number of electrodes along with different topology support which can be configured in different possible ways depending upon the brain region of interest. Power spectral density (PSD) is one of the possible feature extraction methods to identify differences in the brain electrophysiological processing. Table I presents the different EEG bands with their brain functioning. These are discussed in detail in the upcoming sections of this paper.

The rest of the paper is organized as follows: Section II describes background and related work. Proposed method and simulations are presented in Section III. Section IV highlights results and discussion. Conclusion is drawn in Section V.

\section{BACKGROUND AND RELATED WORK}

\section{A. Neurological Disorders (ND)}

Any disorder related to body nervous system comes under the category of neurological disorder. Functional, structural or biochemical abnormalities in the brain circuit or other nerves shows multiple symptoms. While few symptoms are related to physiological changes others are related to neurocognitive functions. There are various forms of mental health disorders and overlapping of symptoms making these disorders hard to diagnose. Figure 1 shows the affected brain regions in neurological disorder.

Few categories of neurological disorders are discussed below.

1) Anxiety disorder: Presence of excessive and persistent fear, worry and anxiety which creates behavioural disturbances are the characteristics of anxiety disorders.

2) Bipolar and related disorder: The characteristics shown by bipolar disorder is mood swing along with changes in physiological activity and energy levels

3) Neurodevelopmental disorder: Group of disorders which are related to the dysfunction of the central nervous system comes under neurodevelopmental disorders

4) Impulse control and conduct disorder: When a person has inability to control emotions and behaviours, he may take certain actions which may harm the individual or others surrounding the subject

5) Substance-related and addictive disorder: Addiction due to the consumption of substances falls under Substance-Related and Addictive Disorders.

6) Major depressive disorder: Mood disorder that causes a persistent feeling of sadness and loss of interest.

7) Internet gaming disorder: When excessive use of Internet or related activites especially online social networking sites and games start to interfere with daily life of subjects.

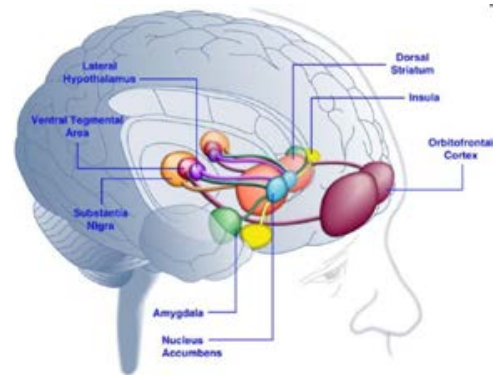

Fig. 1. Affected brain regions in neurological disorders

There are other psychological disorders, which are generated due to altered neurodevelopmental or neuromechanical patterns. It draws behavioral symptoms different than normal ones which impact multiple areas of life. Some experiences erratic thought patterns, mood swing, lack of interest in socializing, lack of empathy, reality and fantasy understanding inability, or a seeming lack of control may have mental disorder. There could be many more symptoms besides these to define mental disorder presence, hence more detailed analysis is required to confirm. WHO does encourages researchers around the world to find evidences and unveil the solution for such addictions. In most or even all addictions or disorder there are few common characteristics like: involving more in addiction than normal, difficulty in quitting, cravings for addiction cause and suffering adverse social consequences due to change in the social behavior as a result of addiction.

\section{B. Major Depressive Disorder (MDD)}

Researchers performed many studies to differentiate brain waves pattern in healthy and depression subjects. Abnormal 
brain activities observed in all studies in some percent of subjects. Lee et al. [10] indicated alpha wave found to be lower in depression group as compared to healthy controls. In [10], alpha band was chosen as region of interest across all the channels to see the features differences where 11 channels showed lower power calculated via mean and standard deviation features. Altogether, they [10] calculated two features for all 32 channels for alpha-1 and alpha-2 waves which were good selected feature for their study.

Hosseinifard et al. [7] extracted five different features from each of the 19 channels, including PSD, DFA, Higuchi fractal dimension, correlation dimension and Lyapunov exponent to find out best feature suitable for their classification algorithm. Trails suggested that GP fractal dimension (GPFD) performed best among the group of selected features with accuracy $83.3 \%$.

Liao et al. [14] showed different montages band power, coherence, GPFDwere selected based on their analysis and relevance check. Using two of the extracted features modified band-pass filtering like CSP and PCA had been applied. The results revealed that kernel eigen filter bankCSP (KEFB-CSP) showed the best feature selection. Authors [14] had used k-NN classifier to define accuracy. Based on KEFB-CSP on temporal region classification accuracy of 81.23\% was achieved.

\section{Internet Gaming Disorder(IGD)}

IGD is related to playing video games in excessive amount over internet which interferes daily life of person and causing relapsing and cultivating the addictive behavior. Behavior of gaming addicts is to get feelings of pleasure and reward in the virtual world. They willingly relinquish sleep, food and physical contact with other human beings or society. Literature [9] reveals that characteristics of both behavioral addiction model of substance use disorders and IGD shows the features of ddysfunctional stress circuits, sensitization, and desensitization. Online games especially MMORPGs are massively prevalent in social networks [8]. There was a debate among researchers about diagnosing internet addiction with IGD as best clinical term, but in last decade IGD got attraction among researchers to further study this internet addiction subtype [19]. Loss of control over gaming is identified as one of the symptom [15-16] and it is argued as impulse control disorder by others [17] or obsessivecompulsive disorder in part by others [18].

Studies have indicated that, symptoms in IGD are similar to major depressive disorder. Subject fails to get enough courage to stop himself getting into activities causing addiction. Research community has categorized the various symptoms of IGD into nine characteristics depicted in Figure 2. Common symptoms are withdrawal symptoms and loss of interest in social life. Duven et al. [3] created virtual game environment to elicit cravings for game and recorded the EEG signals. During this ERP for 30 samples, 15 were pathological extensive game player and other 15 were casual player. From the raw data, latency and peak amplitude of each channel were extracted as features [3]. Authors [3] indicated that P300 was attenuated in case of IGD patients compared to HC. Also longer latencies for N100 was observed.

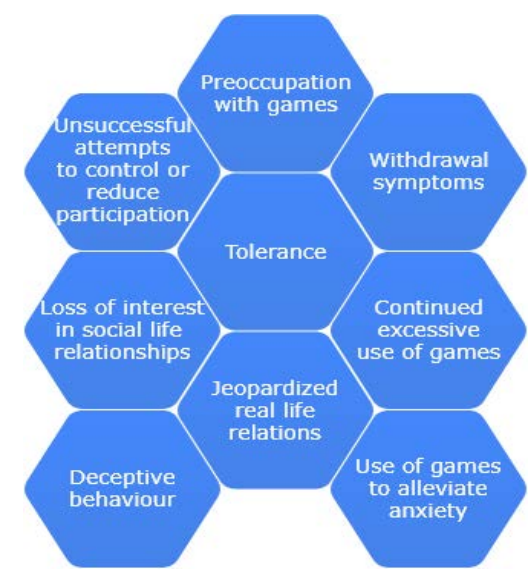

Fig. 2. Most common IGD symptoms.

The latency and amplitude were selected for observation under study performed by Zhu et al. [20]. 102 subjects EEG recording were done. Based on standard IA score the subjects were categorized into 3 groups, which showed different state on the selected features. Latency in $m s$ and peak amplitude in $\mu V$ were compared and result showed latency was high for P300 ERP and amplitude was recorded as low. Based on these features further comparison was done after treatment of subjects.

\section{Simulation MODEL}

In this study, an open source tool box called EEGLAB provided by SCCN lab [2], running under the cross platform MATLAB environment is used for EEG data processing and analysis. Various steps in EEG data analysis are: (a) signal pre-processing to eliminate noises; (b) feature extraction; (c) feature selection; and (d) correlation.

Figure 3 presents the different electrodes are placed in one of the standard topology, EEG sensors (either conductor based or dry electrode based) record the changes in electrical activity due to several millions of neurons functioning in brain as potential.

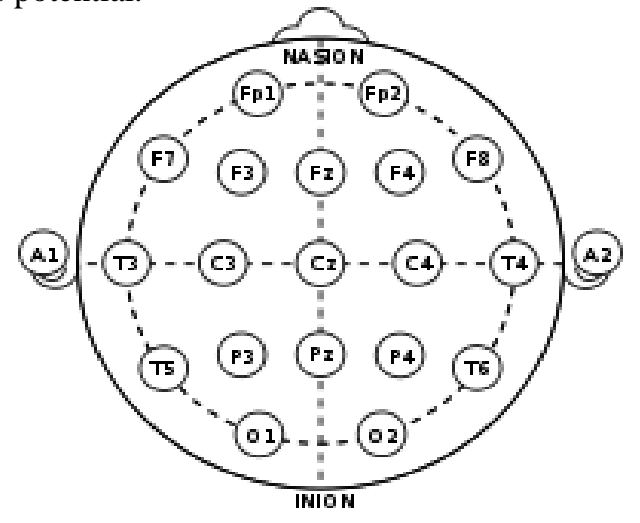

Fig. 3. Standard topology for 19 electrodes.

Like most neurological disorders, it's not possible to isolate an exact cause of IGD. Some evidence suggests that if subject is suffering from IGD his brain makeup is like those that suffer from MDD. Thus, MDD patient's EEG recordings are studied to identify the pattern mentioned as done in [22] for IGD subjects. 


\section{A. Data Source}

EEG recording data for this study has been acquired from the experiment by Wajid et al. [21]. The study shows the rrecruitment of subjects which involved two groups of participants from Hospital Universiti Sain Malaysia (HUSM): (1) 33 depression patients with mean age 40 (16 males and 17 females; and (2) 19 healthy control subjects with mean age 38 (9 males and 10 females). The MDD patients met diagnostic criteria for depression as described in the DSM-IV [23].

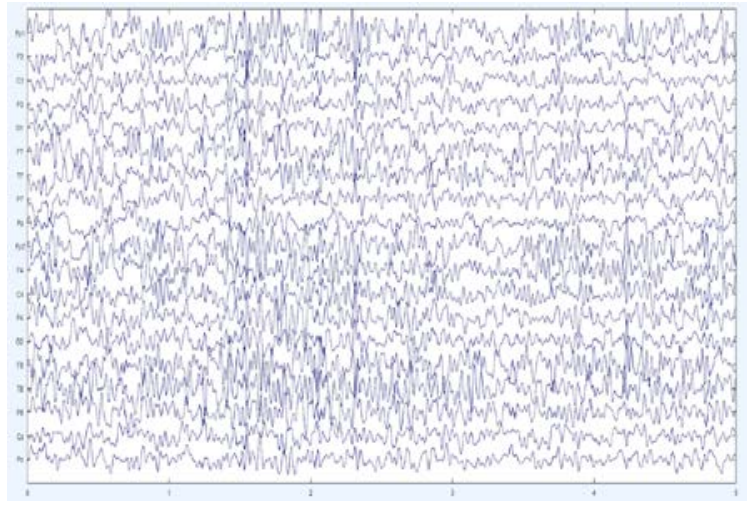

Fig. 4. 19 channels EEG recording.

EEG data was recorded using a 19-channel electro-gel, sensors placed over the scalp. Figure 4 shows the 19 channels EEG recording. Standard 19 channel configuration was used for the placement of 10-20 electrodes over the scalp, named as Fp1, F3, C3, P3, O1, F7, T3, T5, Fz, Fp2, F4, C4, P4, O2, F8, T4, T6, Cz and Pz, which have been standardize with a link-ear (LE) reference. The analogue EEG signals have been amplified and digitized at 256 samples per second and saved in a database.

Data acquisition for experiment for EEG involved two different physiological conditions; viz. EEG recordings during eyes closed (EC) and eyes open (EO) for 5 min each. During EO condition the participants have been instructed to sit relaxed with no eye movement. To avoid drowsiness the studied participants have been interrupted by a beep sound after few intervals. On the other hand, the ERP recordings involved an experiment called the 3-stimulus visual oddball task [24]. During this task, individual responses have been recorded for three different types of stimuli: (a) the target (a blue-colored circle with a radius of $5 \mathrm{~cm}$ ); (b) the distractor (a checker board of similar size as the target); and (c) the standard (a blue-colored circle with a radius of $2.5 \mathrm{~cm}$ ).

\section{B. Data Processing}

Pre-processing of EEG signal is an essential and important step in EEG signal analysis. It helps in removing the unwanted artefacts from the raw signal and make it suitable for further processing. Raw EDF files for MDD patients and control subjects have been uploaded in EEGLAB and saved in MATLAB set files, which can be used in the MATLAB code. All EEG signals were re-referenced using EEGLAB; converting data from fixed reference (earlobe or etc.) to average reference is advocated by some researchers, particularly when the electrode montage covers nearly the whole head (as in some high-density montages).

$$
V_{i}^{C A R}=V_{i}^{E R}-\frac{1}{n} \sum_{j=1}^{n} V_{j}^{E R}
$$

Where $V_{i}^{E R}$ is the potential difference between the $i^{\text {th }}$ electrode and the reference electrodes and $n$ is the number of electrodes in this study (here, $n=19$ ). Figure 5 shows the spectral information of raw signal for one of the MDD patient.

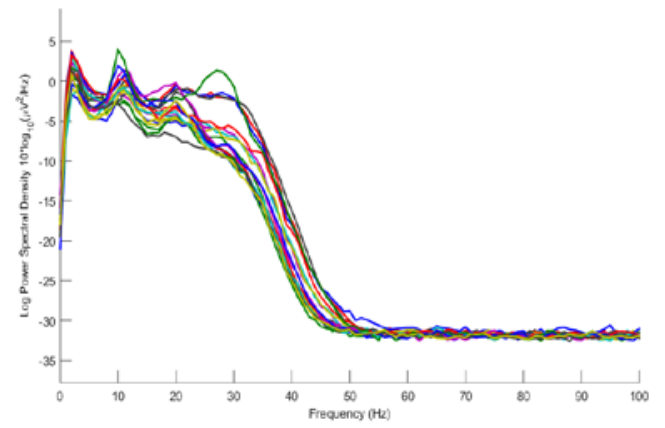

Fig. 5. Power spectral density for raw dataset.

In general, the EEG data are concealed under different types of noises such as generated by human and machine having motors around them. Usually, non-experimental activities by human activities including eye movements, eye blinks, muscular or heart activities, produce non-EEG data in the recordings. Before performing data analysis, EEG datasets were passed through a high pass filter for frequency above $0.5 \mathrm{HZ}$ and low pass filter for frequency below $70 \mathrm{~Hz}$.

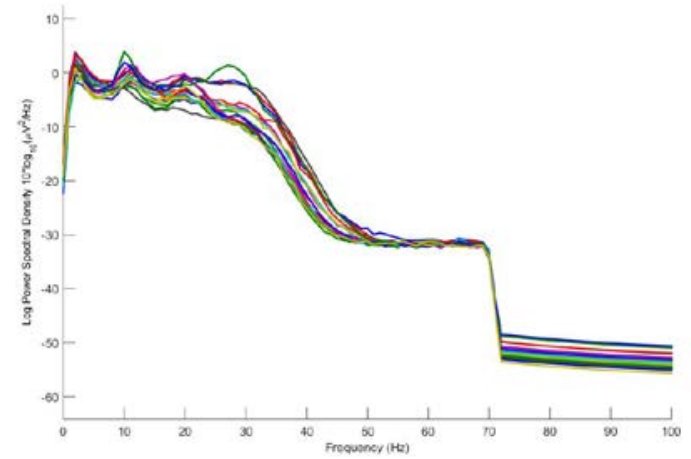

Fig. 6. Power spectral density after pre-processing.

Also, a $50 \mathrm{~Hz}$ notch filter (band stop filter having narrow stop band) is applied to all EEG signals to remove power line noise. Figure 6 shows the refined signal after pre-processing with higher frequency attenuated.

\section{Feature extraction}

After noise removal and normalization, the EEG signal is converted into frequency domain and spectral information is extracted from it. Different EEG signal bands are to be extracted using the discrete wavelet transform (DWT) on raw EEG signal. The signal is passed through series of filters up to level 5 to get the desired frequency bands; which is of our interest in EEG analysis. This decomposition is repeated to increase the frequency resolution and the approximation coefficients decomposed with high and low pass filters and then down-sampled. Figure 7 shows the power across the bands for MDD patients for all 19 channels. 


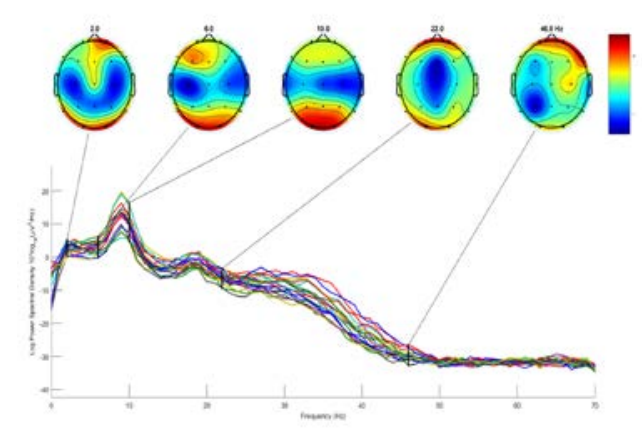

Fig. 7. Power spectra for all the bands for MDD patient

Here, we have focused on absolute power feature which is a basic feature of frequency domain and a good indicator of signal strength of particular EEG band, which can give information on the brain functions during an event or epoch time. Selected feature, an absolute power is determined for each band and, then mean absolute power is derived from all the MDD samples for eyes closed conditions. Similarly, absolute power is calculated for normal subjects for each band and mean absolute power for all normal subjects is computed for eyes closed condition. Relevant values have been stored in a data structure inside the MATLAB code. Table II shows the mean absolute power values from the experimentation. Figure 8 shows the distribution of power across the bands for control subjects for all 19 channels with scalp locations.

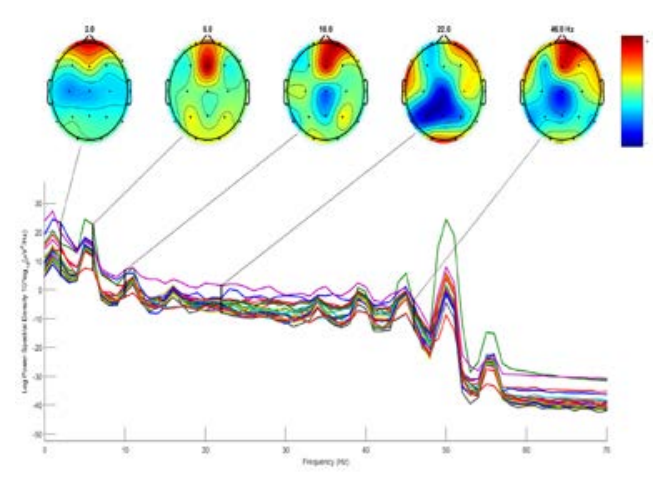

Fig. 8. Power spectra for all bands for normal participant

Same steps are repeated for the samples with eye open conditions for MDD patients and normal subjects and the values were stored in different structures in the code. Captured data has been analyzed.

\section{RESUlTS AND DISCUSSION}

We found reduced beta power in MDD patients as compared to normal subjects in EC conditions, at least in the prefrontal region of brain responsible for the cognitive and logical functions. Figure 9 highlights the difference in power for beta band between MDD patients and controls. No significant changes are observed in the parietal and occipital regions. Right temporal lobe also showed slow beta power, mostly used for the understanding and memorizing the things.

Additionally, the gamma waves found greatly reduced across all channels indicating less activities or brain functions in MDD patients in eyes closed conditions.

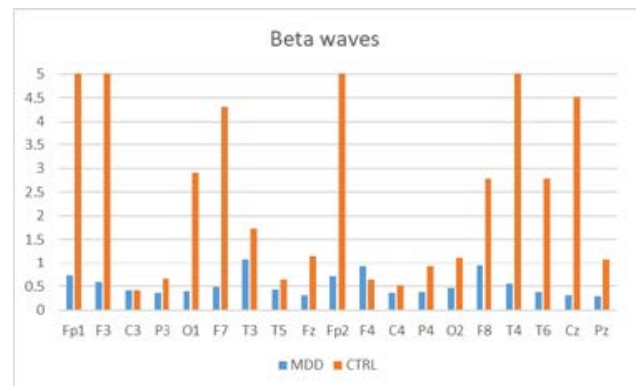

Fig. 8. Beta power for all channels in MDD patients with eyes closed.

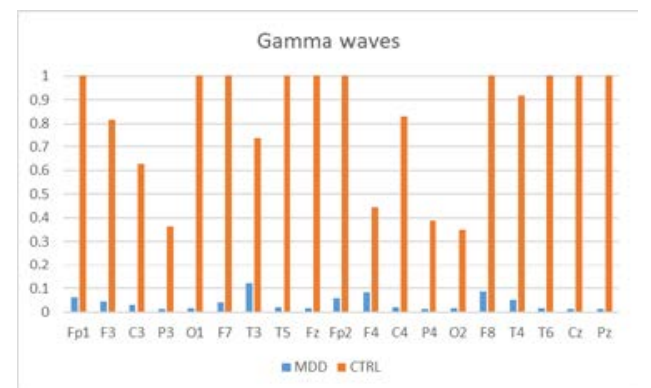

Fig. 9. Comparison of gamma power for all channels with eyes closed.

With EO conditions, in general, reduced power for all the bands was observed except beta band. Beta waves found to have higher amplitude for frontal region, whereas for other channels it was like normal subjects. This is perhaps in coherence with the continuous thoughts in the back of mind in MDD patients, may be related to the cause of depression.

\section{CONCLUSION}

Results obtained through this study shows reduced power in beta waves and gamma oscillations in MDD patients in resting-state eye closed conditions, which is similar to findings for IGD subjects as depicted in [22]. We also found that across all the bands mean absolute power is greatly reduced in the prefrontal region of the brain. Reduced beta power in frontal region amounts to impulse control and changes in the social behaviour of the subjects. Authors [22] also focused on frontal region, this can relate to the insomnia and feeling sad conditions in IGD patients. Due to the similar symptoms for MDD patients and IGD subjects, high frequency waves can be a biomarker for IGD predictions.

The results presented in this paper will opens the door for further studies to identify predictive EEG and ERP based biomarkers for IGD patients. Unfortunately, the diagnosis and treatment for IGD patients is a difficult task, which is still based on subjective assessments and treatment is done through trial and error approach. Though these assessments provide good indications but have many external factors affecting the quality of results obtained from these.

The current research can be extended in future studies to perform similar experiments with IGD subjects and find the optimal values for the difference in high frequency power between IGD subjects and normal participants. Further, machine learning techniques can be applied to classify the IGD subjects based on these bio markers from their EEG recordings. 
TABLE II

Absolute Power For All Bands In Eye Closed Condition

\begin{tabular}{|c|c|c|c|c|c|c|c|c|c|c|c|c|c|c|c|c|c|c|c|}
\hline \multirow{2}{*}{$\begin{array}{l}\text { Channels } \\
\text { Bands } \downarrow\end{array}$} & Fp1 & F3 & C3 & P3 & 01 & F7 & T3 & T5 & Fz & Fp2 & F4 & C4 & P4 & 02 & F8 & T4 & T6 & $\mathrm{Cz}$ & $\mathbf{P z}$ \\
\hline & \multicolumn{19}{|c|}{ MDD patients } \\
\hline Alpha & 1.91 & 1.88 & 1.16 & 2.45 & 5.40 & 1.73 & 1.54 & 3.98 & 1.94 & 1.98 & 1.97 & 1.14 & 2.29 & 5.33 & 1.87 & 1.46 & 2.89 & 1.17 & 2.17 \\
\hline Beta & 0.74 & 0.60 & 0.41 & 0.37 & 0.40 & 0.49 & 1.09 & 0.44 & 0.31 & 0.72 & 0.93 & 0.37 & 0.39 & 0.47 & 0.95 & 0.56 & 0.38 & 0.32 & 0.30 \\
\hline Gamma & 0.06 & 0.04 & 0.03 & 0.01 & 0.02 & 0.04 & 0.12 & 0.02 & 0.02 & 0.06 & 0.08 & 0.02 & 0.01 & 0.02 & 0.09 & 0.05 & 0.02 & 0.01 & 0.01 \\
\hline Theta & 2.02 & 2.43 & 1.93 & 2.23 & 3.39 & 2.37 & 2.26 & 2.80 & 2.22 & 2.36 & 2.38 & 2.03 & 2.09 & 3.28 & 2.44 & 2.15 & 2.38 & 2.22 & 2.23 \\
\hline \multirow[t]{2}{*}{ Delta } & 4.10 & 2.31 & 1.33 & 1.83 & 3.37 & 3.86 & 2.43 & 2.62 & 2.49 & 3.86 & 2.55 & 1.42 & 1.74 & 3.39 & 4.26 & 2.07 & 1.84 & 1.92 & 2.05 \\
\hline & \multicolumn{19}{|c|}{ "Normal Subjects } \\
\hline Alpha & 21.86 & 15.83 & 5.01 & 4.95 & 16.22 & 15.25 & 6.49 & 6.30 & (6.48 & 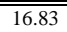 & 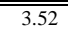 & 4.15 & 5.70 & $\begin{array}{l}11.30 \\
\end{array}$ & 3.99 & 17.30 & $\begin{array}{ll}6.78 \\
\end{array}$ & 5.37 & 4.47 \\
\hline Beta & 6.99 & 6.35 & 0.43 & 0.67 & 2.91 & 4.31 & 1.73 & 0.65 & 1.15 & 5.04 & 0.65 & 0.52 & 0.93 & 1.12 & 2.79 & 6.69 & 2.79 & 4.51 & 1.08 \\
\hline Gamma & 1.05 & 0.82 & 0.63 & 0.36 & 2.36 & 1.37 & 0.74 & 2.42 & 2.17 & 1.40 & 0.45 & 0.83 & 0.39 & 0.35 & 1.96 & 0.92 & 1.89 & 1.38 & 1.66 \\
\hline Delta & 265.50 & 46.24 & 13.75 & 4.91 & 215.48 & 388.75 & 20.96 & 8.14 & 18.97 & 82.18 & 5.97 & 6.47 & 10.03 & 121.86 & 16.06 & 58.44 & 16.22 & 15.25 & 12.83 \\
\hline
\end{tabular}

\section{REFERENCES}

[1] URL: https://venturebeat.com/2018/04/30/newzoo-global-gamesexpected-to-hit-180-1-billion-in-revenues-2021/ visited on $12^{\text {th }}$ May, 2019.

[2] Delorme, A., Makeig, S., 2004. Eeglab: an open source toolbox for analysis of single-trial eeg dynamics including independent com-ponent analysis. Journal of Neuroscience Methods 134 , 9

21

URL:http://www.sciencedirect.com/science/article/pii/S01650270030 03479, doi:https://doi.org/10.1016/j.jneumeth.2003.10.009.

[3] Duven, E., Mller, K., Beutel, M., Wlfling, K., 2014. Altered reward processing in pathological computer gamers erp-results from a seminaturalgaming-design. Brain and Behavior 5. doi:10.1002/brb3.293.

[4] Feigin, V.L., Abajobir, A.A., Abajobir, A.A.a., 2017. Global, regional, and national burden of neurological disorders during 19902015: asystematic analysis for the global burden of disease study 2015. The Lancet. Neurology 16, 877-897. doi:10.1016/S14744422(17)30299-5

[5] Gentile, D.A., Bailey, K., Bavelier, D., Brockmyer, J.F., Cash, H., Coyne, S.M., Doan, A., Grant, D.S., Green, C.S., Griffiths, M., Markle,T., Petry, N.M., Prot, S., Rae, C.D., Rehbein, F., Rich, M., Sullivan, D., Woolley, E., Young, K., 2017. Internet gaming disorder in childrenand adolescents. Pediatrics 140, S81-S85. URL:https://pediatrics.aappublications.org/content/140/Supplement_2 /S81,doi:10.1542/peds.2016-

1758H,arXiv:https://pediatrics.aappublications.org/content/140/Suppl ement2/S81.full.pd f.

[6] Gurau, O., Bosl, W.J., Newton, C.R., 2017. How useful is electroencephalography in the diagnosis of autism spectrum disorders and thedelineation of subtypes: A systematic review. Frontiers in Psychiatry 121. URL:https://www.frontiersin.org/article/10.3389/fpsyt.2017.00121, doi:10.3389/fpsyt.2017.00121.

[7] Hosseinifard, B., Moradi, M.H., Rostami, R., 2013. Classifying depression patients and normal subjects using machine learning techniques andnonlinear features from eeg signal. Computer Methods and Programs in Biomedicine 109, 339 - 345. URL:http://www.sciencedirect.com/science/article/pii/S01692607120 02507, doi:https://doi.org/10.1016/j.cmpb.2012.10.008.

[8] Kimberly Young (2009) Understanding Online Gaming Addiction and Treatment Issues for Adolescents, The American Journal of Family Therapy, 37:5, 355-372, DOI: 10.1080/01926180902942191

[9] Behavioral addictions: an overview. Karim R, Chaudhri P J Psychoactive Drugs. 2012 Jan-Mar; 44(1):5-17

[10] Kan, D.P.X., Lee, P.F., 2015. Decrease alpha waves in depression: An electroencephalogram(eeg) study, in: 2015 International Conference onBioSignal Analysis, Processing and Systems (ICBAPS), pp. 156161. doi:10.1109/ICBAPS.2015.7292237.

[11] Kapur,M.,Tuteja,A.,Prasad,S.,Bawankule,N.,2017.Online gaming in india: Reaching a new pinnacle. https://assets.kpmg/content/dam/kpmg/in/pdf/2017/05/onlinegaming.pdf.

[12] Kuss, D., Griffiths, M., 2012. Internet gaming addiction: a systematic review of empirical research. International Journal of Mental Health andAddiction 10, 278-296. URL:http://irep.ntu.ac.uk/id/eprint/16968/, doi:10.1007/s11469-0119318-5.

[13] Leonard, J., 2018. ”what is gaming disorder?.” medical news today. https://www.medicalnewstoday.com/articles/322478.php.

[14] Liao, S., Wu, C., Huang, H., Cheng, W., Liu, Y., 2017. Major depression detection from EEG signals using kernel eigen-filter-bank $\begin{array}{llll}\text { commonspatial } & \text { patterns. } & \text { Sensors } & 1385 .\end{array}$ URL:https://doi.org/10.3390/s17061385, doi:10.3390/s17061385.

[15] Young KS. Caught in the Net. New York, NY: Wiley (1998)

[16] Young K. Internet addiction: diagnosis and treatment considerations. J Contemp Psychother (2009) 39(4):241-6. doi:10.1007/s10879-0099120-x

[17] Aboujaoude E. Problematic Internet use: an overview. World Psychiatry (2010) 9:85-90. doi:10.1002/j.2051-5545.2010.tb00278.x

[18] Dell'Osso B, Altamura C, Allen A, Marazziti D, Hollander E. Epidemiological and clinical updates on impulse control disorders: a critical review. Eur Arch Psychiatry Clin Neurosci (2006) 256:46475. doi:10.1007/s00406-006-0668-0

[19] American Psychiatric Association. Diagnostic and Statistical Manual of Mental Disorders: DSM-5. Washington, DC: American Psychiatric Association (2013)

[20] Zhu, T.M., Li, H., Jin, R.J., Zheng, Z., Luo, Y., Ye, H., Zhu, H.M., 2012. Effects of electroacupuncture combined psycho-intervention oncognitive function and event-related potentials p300 and mismatch negativity in patients with internet addiction. Chinese journal of integrativemedicine 18, 146-51. doi:10.1007/s11655-012-0990-5.

[21] Mumtaz, Wajid \& Malik, Aamir \& mohd yasin, mohd azhar \& Xia, Likun. (2015). Review on EEG and ERP predictive biomarkers for major depressive disorder. Biomedical Signal Processing and Control. 22. 85-98. 10.1016/j.bspc.2015.07.003.

[22] Choi, Jung-Seok \& Park, Su Mi \& Lee, Jaewon \& Yeon Hwang, Jae \& Yeon Jung, Hee \& Choi, Sam-Wook \& Kim, Dai-Jin \& Oh, Sohee \& Lee, Jun-Young. (2013). Resting-state beta and gamma activity in Internet addiction. International journal of psychophysiology : official journal of the International Organization of Psychophysiology. 89. 10.1016/j.ijpsycho.2013.06.007.

[23] A.P. Association, Diagnostic and Statistical Manual-IV (1994), American Psychiatric Association, Washington, DC, 1994

[24] J. Polich, J.R. Criado, Neuropsychology and neuropharmacology of P3a and P3b, Int. J. Psychophysiol. 60 (2006) 172-185. 